\title{
Article \\ On the Admissibility of the Fixed Points Set of a Mapping with Respect to Another Mapping
}

\section{Bessem Samet}

check for updates

Citation: Samet, B. On the Admissibility of the Fixed Points Set of a Mapping with Respect to Another Mapping. Mathematics 2021, 9, 1981. https://doi.org/10.3390/ math9161981

Academic Editors: Óscar Valero Sierra and Hsien-Chung Wu

Received: 9 July 2021

Accepted: 14 August 2021

Published: 19 August 2021

Publisher's Note: MDPI stays neutral with regard to jurisdictional claims in published maps and institutional affiliations.

Copyright: (C) 2021 by the author. Licensee MDPI, Basel, Switzerland. This article is an open access article distributed under the terms and conditions of the Creative Commons Attribution (CC BY) license (https:/ / creativecommons.org/licenses/by/ $4.0 /)$.
Department of Mathematics, College of Science, King Saud University, P.O. Box 2455, Riyadh 11451, Saudi Arabia; bsamet@ksu.edu.sa

Abstract: Let $(M, \delta)$ be a metric space, $f: M \rightarrow M$, and $g: M \rightarrow[0,+\infty)$. In this paper, we obtain sufficient conditions under which the set of fixed points of $f$ is $g$-admissible, i.e., $\operatorname{Fix}(f) \neq \varnothing$ and $\operatorname{Fix}(f) \subset g^{-1}(\{0\})$. Some special cases of our main results are discussed and some examples are given.

Keywords: fixed points set; $g$-admissible; $\mu$-continuous; $\mu$-regular; $\mu$-Cauchy; $\mu$-complete; picard sequence

\section{Introduction}

Let $(M, \delta)$ be a metric space, $f: M \rightarrow M$, and $g: M \rightarrow[0,+\infty)$. In this paper, we are interested in obtaining sufficient conditions on $(M, \delta), f$, and $g$, ensuring that

$$
\left\{\begin{array}{l}
\operatorname{Fix}(f) \neq \varnothing, \\
\operatorname{Fix}(f) \subset g^{-1}(\{0\}),
\end{array}\right.
$$

where $\operatorname{Fix}(f)$ denotes the set of fixed points of $f$. In this case, we say that Fix $(f)$ is $g$-admissible.

The main motivation for studying problems of type (1) comes from the theory of partial metric spaces (see e.g., [1,2]) and essentially from Ioan A. Rus's paper [3]. Namely, let $(M, \rho)$ be a complete partial metric space and $f: M \rightarrow M$ be a given mapping. Suppose that there exists $0<\sigma<1$ such that

$$
\rho(f(y), f(z)) \leq \sigma \rho(y, z),
$$

for all $y, z \in M$. Then (see [2]) $f$ has a unique fixed point $z^{*} \in M$. Moreover, $\rho\left(z^{*}, z^{*}\right)=0$. Consider now the mapping $\delta_{\rho}: M \times M \rightarrow[0,+\infty)$ defined by

$$
\delta_{\rho}(y, z)=2 \rho(y, z)-\rho(y, y)-\rho(z, z), \quad y, z \in M \text {. }
$$

Then (see [4]) $\left(M, \delta_{\rho}\right)$ is a complete metric space. Moreover, (2) is equivalent to

$$
\delta_{\rho}(f(y), f(z))+\rho(f(y), f(y))+\rho(f(z), f(z)) \leq \sigma\left(\delta_{\rho}(y, z)+\rho(y, y)+\rho(z, z)\right),
$$

for all $y, z \in M$. In [3], Ioan A. Rus proposed to study the class of mappings $f$ satisfying the more general condition

$$
\delta(f(y), f(z))+g(f(y))+g(f(z)) \leq \sigma(\delta(y, z)+g(y)+g(z))
$$

for all $y, z \in M$, where $\delta$ is a metric on $M$ and $g: M \rightarrow[0,+\infty)$ is a given mapping. In [5], it was shown that, if $(M, \delta)$ is complete, $g$ is lower semi-continuous, and $f$ and $g$ satisfy (3), then $f$ admits a unique fixed point $z^{*} \in M$. Moreover, $g\left(z^{*}\right)=0$. Some extensions and generalizations of the obtained result in [5] can be found in [6-12] (see also the references therein). Some recent results related to fixed point theory in partial metric spaces can be found in [13-16] (see also the references therein). 
This work is concerned with the study of new classes of mappings $f: M \rightarrow M$ for which we derive $g$-admissibility results. The next section of this paper is devoted to some definitions and preliminaries. In Section 3, we present and prove our main results. In Section 4, we investigate some special cases and provide some examples.

\section{Preliminaries}

Let $(M, \delta)$ be a metric space and $\mu: M \times M \rightarrow \mathbb{R}$. Let $H$ be the set of functions $h:[0,+\infty) \rightarrow[0,+\infty)$ satisfying:

(H1) $h$ is nondecreasing,

(H2) $\sum_{i=0}^{+\infty} h^{i}(s)<+\infty$ for all $s>0$, where $h^{i}$ is the ith iterate of $h$.

Throughout this paper, $\mathbb{N}$ denotes the set of natural numbers. We recall below some notions introduced in [17] (see also [18]).

Definition 1. A sequence $\left\{z_{n}\right\} \subset M$ is $\mu$-regular, if $\mu\left(z_{n}, z_{n+1}\right) \geq 1$ for all $n \in \mathbb{N}$.

Definition 2. A sequence $\left\{z_{n}\right\} \subset M$ is $\mu$-Cauchy, if $\left\{z_{n}\right\}$ is $\mu$-regular and $\left\{z_{n}\right\}$ is a Cauchy sequence.

Definition 3. The metric space $(M, \delta)$ is $\mu$-complete, if every $\mu$-Cauchy sequence in $M$ is convergent to an element of $M$.

Definition 4. A mapping $f: M \rightarrow M$ is $\mu$-continuous, if for every $\mu$-regular sequence $\left\{z_{n}\right\} \subset M$,

$$
\lim _{n \rightarrow+\infty} \delta\left(z_{n}, z\right)=0, z \in M \Longrightarrow \lim _{n \rightarrow+\infty} \delta\left(f\left(z_{n}\right), f(z)\right)=0 .
$$

Definition 5 (see [8]). Let $f: M \rightarrow M$ and $g: M \rightarrow[0,+\infty)$. The set $\operatorname{Fix}(f)$ is said to be $g$-admissible, if it satisfies (1).

Some examples on the above notions are given below.

Example 1. Let $M=\mathbb{R}$ and $\mu: M \times M \rightarrow \mathbb{R}$ be the mapping defined by

$$
\mu(t, s)=\sin ^{2}\left(\frac{\pi t}{2}\right)+\sin ^{2}\left(\frac{\pi s}{2}\right), \quad(t, s) \in M \times M .
$$

Let $\left\{z_{n}\right\} \subset M$ be the sequence defined by

$$
z_{n}=n, \quad n \in \mathbb{N} .
$$

Then, for all $n \in \mathbb{N}$,

$$
\begin{aligned}
\mu\left(z_{n}, z_{n+1}\right) & =\sin ^{2}\left(\frac{n \pi}{2}\right)+\sin ^{2}\left(\frac{(n+1) \pi}{2}\right) \\
& =\sin ^{2}\left(\frac{n \pi}{2}\right)+\cos ^{2}\left(\frac{n \pi}{2}\right) \\
& =1
\end{aligned}
$$

which shows that $\left\{z_{n}\right\}$ is $\mu$-regular.

Example 2. Let $M=C([0,1])$ and $\delta$ be the metric on $M$ defined by

$$
\delta(f, g)=\max _{0 \leq t \leq 1}|f(t)-g(t)|, \quad f, g \in M .
$$


Let $\mu: M \times M \rightarrow \mathbb{R}$ be the mapping defined by

$$
\mu(f, g)=\int_{0}^{1} f(s) g(s) d s, \quad f, g \in M .
$$

Consider the sequence $\left\{z_{n}\right\} \subset M$ defined by

$$
z_{n}(t)=\exp \left(\frac{t}{n+1}\right), \quad 0 \leq t \leq 1
$$

Since $\left\{z_{n}\right\}$ is a convergent sequence (it converges uniformly to $z(t)=1$ ), then $\left\{z_{n}\right\}$ is a Cauchy sequence. Moreover, for all $n \in \mathbb{N}$,

$$
\mu\left(z_{n}, z_{n+1}\right)=\int_{0}^{1} \exp \left(\frac{t}{n+1}\right) \exp \left(\frac{t}{n+2}\right) d t \geq 1 .
$$

Then $\left\{z_{n}\right\}$ is $\mu$-regular. Consequently, $\left\{z_{n}\right\}$ is a $\mu$-Cauchy sequence.

Example 3. Let $M=(0,+\infty)$ and $\delta=|\cdot|$ (i.e., $\delta(y, z)=|y-z|$ for all $y, z \in M)$. Obviously $(M, \delta)$ is not complete. Let $\mu: M \times M \rightarrow \mathbb{R}$ be the mapping defined by

$$
\mu(t, s)= \begin{cases}1 & \text { if } t, s \geq 1 \\ 0 & \text { if } 0<t<1 \text { or } 0<s<1\end{cases}
$$

Consider a $\mu$-Cauchy sequence $\left\{z_{n}\right\} \subset M$. Due to the $\mu$-regularity of $\left\{z_{n}\right\}$, by the definition of $\mu$, we deduce that $\left\{z_{n}\right\} \subset \mathcal{M}=[1, \infty)$. Since $(\mathcal{M}, \delta)$ is complete and $\left\{z_{n}\right\}$ is a Cauchy sequence, then $\left\{z_{n}\right\}$ converges to some $z \in \mathcal{M} \subset M$. This shows that $(M, \delta)$ is $\mu$-complete. Consider now the function $f: M \rightarrow M$ defined by

$$
f(t)= \begin{cases}-\ln t & \text { if } 0<t<1 \\ t & \text { if } t \geq 1\end{cases}
$$

Clearly $f$ is not continuous at 1 . However, for any $\mu$-regular sequence $\left\{z_{n}\right\} \subset M$ (recall that in this case $z_{n} \geq 1$ for all $n$ ), if $z_{n} \rightarrow z \in M$, as $n \rightarrow+\infty$, then $z \geq 1$ and $f\left(z_{n}\right)=z_{n} \rightarrow f(z)=z$ as $n \rightarrow+\infty$. This shows that $f$ is $\mu$-continuous.

The proof of the following lemma can be found in [19].

Lemma 1. Let $h \in H$. Then

(i) $h(s)<s$ for all $s>0$,

(ii) $h(0)=0$,

(iii) $h$ is continuous at 0 .

\section{Main Results}

The first main result of this paper is the following.

Theorem 1. Let $(M, \delta)$ be a metric space, $\mu: M \times M \rightarrow \mathbb{R}, f: M \rightarrow M$, and $g: M \rightarrow[0,+\infty)$. Assume that

(i) $(M, \delta)$ is $\mu$-complete,

(ii) $f$ is $\mu$-continuous,

(iii) $\mu(z, f(z)) \geq 1$ for all $z \in M$.

Suppose also that there exists $h \in H$ such that

$$
\mu(y, z)(\delta(f(y), f(z))+g(f(y))+g(f(z))) \leq h(\delta(y, z)+g(y)+g(z)),
$$

for all $y, z \in M$. Then 
(I) $\operatorname{Fix}(f)$ is g-admissible,

(II) For all $z_{0} \in M$, the Picard sequence $\left\{f^{n}\left(z_{0}\right)\right\}$ converges to a fixed point of $f$.

Proof. We first show that

$$
\operatorname{Fix}(f) \subset g^{-1}(\{0\})
$$

If $\operatorname{Fix}(f)=\varnothing$, then (5) is obvious. So, suppose that $\operatorname{Fix}(f) \neq \varnothing$ and let $z \in \operatorname{Fix}(f)$. Taking $y=z$ in (4), we obtain

$$
\mu(z, z)(\delta(f(z), f(z))+g(f(z))+g(f(z))) \leq h(\delta(z, z)+g(z)+g(z)),
$$

that is,

$$
2 g(z) \mu(z, f(z)) \leq h(2 g(z))
$$

Due to (iii), we deduce that

$$
2 g(z) \leq h(2 g(z))
$$

If $g(z)>0$, then by the statement (i) of Lemma 1, we have

$$
h(2 g(z))<2 g(z)
$$

Hence, (6) and (7) lead to a contradiction. Consequently, $g(z)=0$ and (5) follows.

The second step consists in showing that $\operatorname{Fix}(f)$ is a nonempty set. Fix $z_{0} \in M$ and consider the Picard sequence $\left\{z_{n}\right\} \subset M$ defined by

$$
z_{n+1}=f\left(z_{n}\right), \quad n \in \mathbb{N} .
$$

Then, for $n \geq 1$, taking $(y, z)=\left(z_{n-1}, z_{n}\right)$ in (4), we obtain $\mu\left(z_{n-1}, z_{n}\right)\left(\delta\left(f\left(z_{n-1}\right), f\left(z_{n}\right)\right)+g\left(f\left(z_{n-1}\right)\right)+g\left(f\left(z_{n}\right)\right)\right) \leq h\left(\delta\left(z_{n-1}, z_{n}\right)+g\left(z_{n-1}\right)+g\left(z_{n}\right)\right)$, that is,

$$
\mu\left(z_{n-1}, z_{n}\right)\left(\delta\left(z_{n}, z_{n+1}\right)+g\left(z_{n}\right)+g\left(z_{n+1}\right)\right) \leq h\left(\delta\left(z_{n-1}, z_{n}\right)+g\left(z_{n-1}\right)+g\left(z_{n}\right)\right) .
$$

Since by (iii) $\left\{z_{n}\right\}$ is $\mu$-regular, the above inequality leads to

$$
\tau_{n} \leq h\left(\tau_{n-1}\right), \quad n \geq 1,
$$

where

$$
\tau_{n}=\delta\left(z_{n}, z_{n+1}\right)+g\left(z_{n}\right)+g\left(z_{n+1}\right) .
$$

By $(\mathrm{H} 1)$, the above inequality leads to

$$
\tau_{1} \leq h\left(\tau_{0}\right), \quad \tau_{2} \leq h\left(\tau_{1}\right) \leq h^{2}\left(\tau_{0}\right), \quad \tau_{3} \leq h\left(\tau_{2}\right) \leq h^{3}\left(\tau_{0}\right), \cdots,
$$

that is,

$$
\tau_{n} \leq h^{n}\left(\tau_{0}\right), \quad n \geq 1 .
$$

Since $\delta\left(z_{n}, z_{n+1}\right) \leq \tau_{n}$, we deduce that

$$
\delta\left(z_{n}, z_{n+1}\right) \leq h^{n}\left(\tau_{0}\right), \quad n \geq 1 .
$$


Using (11) and the triangle inequality, for $n<m$, it holds that

$$
\begin{aligned}
\delta\left(z_{n}, z_{m}\right) & \leq \sum_{r=n}^{m-1} \delta\left(z_{r}, z_{r+1}\right) \\
& \leq \sum_{r=n}^{m-1} h^{r}\left(\tau_{0}\right) \\
& \leq \sum_{r=n}^{+\infty} h^{r}\left(\tau_{0}\right) .
\end{aligned}
$$

Hence, due to (H2) and the statement (ii) of Lemma 1, we deduce that

$$
\lim _{n, m \rightarrow+\infty} \delta\left(z_{n}, z_{m}\right)=0,
$$

which proves that $\left\{z_{n}\right\}$ is a Cauchy sequence, and then $\left\{z_{n}\right\}$ is $\mu$-Cauchy. Therefore, by (i), we deduce that $\left\{z_{n}\right\}$ converges to some $z^{*} \in M$. Since $\left\{z_{n}\right\}$ is $\mu$-regular and $f$ is $\mu$-continuous (by (ii)), it holds that $\left\{f\left(z_{n}\right)\right\}=\left\{z_{n+1}\right\}$ converges to $f\left(z^{*}\right)$. Then the uniqueness of the limit leads to $z^{*}=f\left(z^{*}\right)$, that is, $z^{*} \in \operatorname{Fix}(f)$. Consequently

$$
\operatorname{Fix}(f) \neq \varnothing \text {. }
$$

Hence, (5) and (12) show that $\operatorname{Fix}(f)$ is $g$-admissible, which proves (I). Finally, (II) follows from the the arbitrary choice of $z_{0}$ and the convergence of $\left\{z_{n}\right\}$ to an element of $\operatorname{Fix}(f)$.

Our next result is the following.

Theorem 2. Let $(M, \delta)$ be a metric space, $\mu: M \times M \rightarrow \mathbb{R}, f: M \rightarrow M$, and $g: M \rightarrow[0,+\infty)$. Assume that conditions (i) and (iii) of Theorem 1, and (4) hold. Moreover, suppose that

(a) $g$ is lower semi-continuous,

(b) For any $\mu$-regular sequence $\left\{u_{n}\right\} \subset M$,

$$
\lim _{n \rightarrow+\infty} \delta\left(u_{n}, u\right)=0, u \in M \Longrightarrow \exists k \in \mathbb{N}: \mu\left(u_{n}, u\right) \geq 1, n \geq k .
$$

Then

(I) $\operatorname{Fix}(f)$ is g-admissible,

(II) For all $z_{0} \in M$, the Picard sequence $\left\{f^{n}\left(z_{0}\right)\right\}$ converges to a fixed point of $f$.

Proof. From the proof of Theorem 1, (5) holds, and the Picard sequence $\left\{z_{n}\right\}$ defined by (8) is $\mu$-regular and converges to some $z^{*} \in M$. By (b), we deduce that there exists $k \in \mathbb{N}$ such that

$$
\mu\left(z_{n}, z^{*}\right) \geq 1, \quad n \geq k
$$

On the other hand, by (H2), the statement (ii) of Lemma 1, and (10), we deduce that

$$
\lim _{n \rightarrow+\infty} \tau_{n}=0
$$

Hence, from (9) (since $\left\{z_{n}\right\}$ is convergent), it follows that

$$
\lim _{n \rightarrow+\infty} g\left(z_{n}\right)=0,
$$

which implies by (a) that

$$
g\left(z^{*}\right) \leq \liminf _{n \rightarrow+\infty} g\left(z_{n}\right)=0
$$

that is,

$$
g\left(z^{*}\right)=0 .
$$


Next, taking $(y, z)=\left(z_{n}, z^{*}\right)$ in (4), using (13) and (15), it holds that

$$
\delta\left(z_{n+1}, f\left(z^{*}\right)\right)+g\left(z_{n+1}\right)+g\left(f\left(z^{*}\right)\right) \leq h\left(\delta\left(z_{n}, z^{*}\right)+g\left(z_{n}\right)\right), \quad n \geq k,
$$

which yields

$$
\delta\left(z_{n+1}, f\left(z^{*}\right)\right) \leq h\left(\delta\left(z_{n}, z^{*}\right)+g\left(z_{n}\right)\right), \quad n \geq k .
$$

Passing to the limit as $n \rightarrow+\infty$ in the above inequality, using the statements (ii) and (iii) of Lemma 1, and (14), we obtain $\delta\left(z^{*}, f\left(z^{*}\right)\right)=0$, that is, $z^{*} \in \operatorname{Fix}(f)$. Hence, by (5), Fix $(f)$ is $g$-admissible.

\section{Special Cases}

Some special cases of our main results are deduced in this section.

Corollary 1. Let $(M, \delta)$ be a complete metric space, $f: M \rightarrow M$, and $g: M \rightarrow[0,+\infty)$. Suppose that there exist $h \in H$ and $F:[0,+\infty) \rightarrow \mathbb{R}$ such that

$$
\delta(f(y), f(z))+g(f(y))+g(f(z)) \leq h(\delta(y, z)+g(y)+g(z))+F(\delta(z, f(y))), \quad y, z \in M .
$$

\section{Suppose also that}

(i) $g$ is lower semi-continuous,

(ii) $F$ is continuous,

(iii) $F(0)=0$.

Then

(I) $\operatorname{Fix}(f)$ is g-admissible,

(II) For all $z_{0} \in M$, the Picard sequence $\left\{f^{n}\left(z_{0}\right)\right\}$ converges to a fixed point of $f$.

Proof. The main idea of the proof consists in showing that $f$ and $g$ satisfy (4) for a judicious choice of the mapping $\mu$. Namely, let us introduce the mapping $\mu: M \times M \rightarrow \mathbb{R}$ defined by

$$
\mu(y, z)=\left\{\begin{array}{lll}
1 & \text { if } & z=f(y) \\
0 & \text { if } & z \neq f(y) .
\end{array}\right.
$$

Obviously, one has

$$
\mu(y, f(y))=1, \quad y \in M .
$$

Let us fix $(y, z) \in M$. If $z \neq f(y)$, then by the definition of $\mu$, (4) follows immediately. If $z=f(y)$, it follows from (16) that

$$
\delta(f(y), f(z))+g(f(y))+g(f(z)) \leq h(\delta(y, z)+g(y)+g(z))+F(0) .
$$

Since $F(0)=0$ and $\mu(y, z)=1$, it holds that

$$
\mu(y, z)(\delta(f(y), f(z))+g(f(y))+g(f(z))) \leq h(\delta(y, z)+g(y)+g(z)) .
$$

Then, in both cases, (4) is satisfied.

Next, let us show that $f$ is $\mu$-continuous. Let $\left\{z_{n}\right\}$ be a $\mu$-regular sequence. By the definition of $\mu$, it holds that $z_{n+1}=f\left(z_{n}\right)$ for all $n \in \mathbb{N}$. Let $u \in M$ be such that $\lim _{n \rightarrow+\infty} \delta\left(z_{n}, u\right)=0$. Taking $(y, z)=\left(z_{n-1}, z_{n}\right)$ in (16), we obtain (since $F(0)=0$ )

$$
\delta\left(z_{n}, z_{n+1}\right)+g\left(z_{n}\right)+g\left(z_{n+1}\right) \leq h\left(\delta\left(z_{n-1}, z_{n}\right)+g\left(z_{n-1}\right)+g\left(z_{n}\right)\right), \quad n \geq 1 .
$$


Since $h \in H$, the above inequality leads to

$$
\lim _{n \rightarrow+\infty} g\left(z_{n}\right)=0 .
$$

Due to the lower semi-continuity of $g$, we deduce that

$$
g(u)=0 .
$$

Hence, taking $(y, z)=\left(z_{n}, u\right)$ in (16), it holds that

$$
\delta\left(f\left(z_{n}\right), f(u)\right) \leq \delta\left(f\left(z_{n}\right), f(u)\right)+g\left(f\left(z_{n}\right)\right)+g(f(u)) \leq h\left(\delta\left(z_{n}, u\right)+g\left(z_{n}\right)\right)+F\left(\delta\left(u, z_{n+1}\right)\right),
$$

for all $n \in \mathbb{N}$. Passing to the limit as $n \rightarrow+\infty$, using the statements (ii) and (iii) of Lemma 1 , the continuity of $F$, and the fact that $F(0)=0$, we obtain

$$
\lim _{n \rightarrow+\infty} \delta\left(f\left(z_{n}\right), f(u)\right)=0
$$

This shows that $f$ is $\mu$-continuous.

Notice that since $(M, \delta)$ is complete, then $(M, \delta)$ is $\mu$-complete. Finally, the desired results follow from Theorem 1 .

Remark 1. The class of mappings $f: M \rightarrow M$ satisfying (16) with $g \equiv 0$ and $F(s)=L s, L \geq 0$, has been introduced in [20] under the name of $(h, L)$-weak contractions. Namely, it was shown that, if $f$ belongs to the class of $(h, L)$-weak contractions, then Fix $(f) \neq \varnothing$ and for all $z_{0} \in M$, the Picard sequence $\left\{f^{n}\left(z_{0}\right)\right\}$ converges to a fixed point of $f$. Clearly, taking $g \equiv 0$ and $F(s)=L s$ in Corollary 1, we refind these results. For additional references related to $(h, L)$-weak contractions and their generalizations, see e.g., $[17,19,21-25]$ and the references therein.

An example supporting Corollary 1 is given below.

Example 4. Let $M=[0,1]$ and $\delta$ be the standard metric on $M$ given by

$$
\delta(y, z)=|y-z|, \quad y, z \in M .
$$

Consider the mappings $f: M \rightarrow M$ and $g: M \rightarrow[0,+\infty)$ defined respectively by

$$
f(y)= \begin{cases}\frac{1}{4} & \text { if } y \in[0,1), \\ 0 & \text { if } y=1,\end{cases}
$$

and

$$
g(y)=\left|4 y^{2}-3 y+\frac{1}{2}\right|, \quad y \in M .
$$

We can check easily that $\operatorname{Fix}(f)=\left\{\frac{1}{4}\right\}$ and $g^{-1}(\{0\})=\left\{\frac{1}{2}, \frac{1}{4}\right\}$. We claim that

$\delta(f(y), f(z))+g(f(y))+g(f(z)) \leq \frac{1}{2}(\delta(y, z)+g(y)+g(z))+\delta(z, f(y)), \quad y, z \in M$.

- Case 1: $0 \leq y, z<1$. In this case, we have

$$
\delta(f(y), f(z))+g(f(y))+g(f(z))=\left|\frac{1}{4}-\frac{1}{4}\right|+g\left(\frac{1}{4}\right)+g\left(\frac{1}{4}\right)=0 .
$$


Then (20) holds.

- Case 2: $0 \leq y<1$ and $z=1$. In this case,

$$
\begin{aligned}
\delta(f(y), f(z))+g(f(y))+g(f(z)) & =\frac{1}{4}+g\left(\frac{1}{4}\right)+g(0) \\
& =\frac{3}{4} \\
& =\delta(z, f(y)) \\
& \leq \frac{1}{2}(\delta(y, z)+g(y)+g(z))+\delta(z, f(y)) .
\end{aligned}
$$

- Case 3: $y=1$ and $0 \leq z<1$. In this case,

$$
\begin{aligned}
\delta(f(y), f(z))+g(f(y))+g(f(z)) & =\frac{3}{4} \\
& =\frac{g(y)}{2} \\
& \leq \frac{1}{2}(\delta(y, z)+g(y)+g(z))+\delta(z, f(y)) .
\end{aligned}
$$

- Case 4: $y=z=1$. In this case,

$$
\begin{aligned}
\delta(f(y), f(z))+g(f(y))+g(f(z)) & =2 g(0) \\
& =1 \\
& =\delta(z, f(y)) \\
& \leq \frac{1}{2}(\delta(y, z)+g(y)+g(z))+\delta(z, f(y)) .
\end{aligned}
$$

Hence, (20) is satisfied in all cases. Notice that (20) is a special case of (16) with

$$
h(s)=\frac{s}{2}, \quad s \geq 0
$$

and

$$
F(s)=s, \quad s \geq 0 .
$$

Then, by Corollary 1, $\operatorname{Fix}(f)$ is g-admissible, and for all $z_{0} \in M$, the Picard sequence $\left\{f^{n}\left(z_{0}\right)\right\}$ converges to a fixed point of $f$. Notice that

$$
\operatorname{Fix}(f)=\left\{\frac{1}{4}\right\} \subset\left\{\frac{1}{2}, \frac{1}{4}\right\}=g^{-1}(\{0\}),
$$

which confirms the results given by Corollary 1.

Corollary 2. Let $(M, \delta)$ be a complete metric space, $f: M \rightarrow M$, and $g: M \rightarrow[0,+\infty)$. Suppose that there exist $\sigma>0$ and $F:[0,+\infty) \times[0,+\infty) \rightarrow[0,+\infty)$ such that

$$
\begin{aligned}
& \delta(f(y), f(z))+g(f(y))+g(f(z)) \\
& \leq F(\delta(y, f(y))+g(y)+g(f(y)), \delta(y, z)+g(y)+g(z))(\delta(z, f(z))+g(z)+g(f(z))) \\
& +\sigma(\delta(y, z)+g(y)+g(z)),
\end{aligned}
$$

for all $y, z \in$ M. Suppose also that

(i) $g$ is lower semi-continuous,

(ii) $F$ is continuous,

(iii) There exists $\lambda_{F}>0$ such that $F(s, s)=\lambda_{F}$ for all $s \geq 0$,

(iv) $\sigma+\lambda_{F}<1$. 


\section{Then}

(I) $\operatorname{Fix}(f)$ is g-admissible,

(II) For all $z_{0} \in M$, the Picard sequence $\left\{f^{n}\left(z_{0}\right)\right\}$ converges to a fixed point of $f$.

Proof. First, we shall prove that $f$ and $g$ satisfy (4) for some $h \in H$, where $\mu$ is defined by (17). Let us fix $(y, z) \in M$. If $z \neq f(y)$, then by the definition of $\mu$, (4) follows immediately. If $z=f(y)$, it follows from (21) that

$$
\begin{aligned}
& \delta(f(y), f(z))+g(f(y))+g(f(z)) \\
& \leq F(\delta(y, z)+g(y)+g(z), \delta(y, z)+g(y)+g(z))(\delta(f(y), f(z))+g(f(y))+g(f(z))) \\
& +\sigma(\delta(y, z)+g(y)+g(z)) .
\end{aligned}
$$

Using (iii), we deduce that

$$
\begin{aligned}
\delta(f(y), f(z))+g(f(y))+g(f(z)) \leq & \lambda_{F}(\delta(f(y), f(z))+g(f(y))+g(f(z))) \\
& +\sigma(\delta(y, z)+g(y)+g(z)),
\end{aligned}
$$

which yields

$$
\left(1-\lambda_{F}\right)(\delta(f(y), f(z))+g(f(y))+g(f(z))) \leq \sigma(\delta(y, z)+g(y)+g(z)) .
$$

Since $1-\lambda_{F}>0$ and $\mu(y, z)=1$, it holds that

$$
\mu(y, z)(\delta(f(y), f(z))+g(f(y))+g(f(z))) \leq h(\delta(y, z)+g(y)+g(z)),
$$

where

$$
h(s)=\frac{\sigma s}{1-\lambda_{F}}, \quad s \geq 0 .
$$

Notice that form (iv), it follows that $h \in H$. Hence, $f$ and $g$ satisfy (4), where $h$ is defined above and $\mu$ is given by (17). Moreover, proceeding as in the proof of Corollary 1 , one can show that $f$ is $\mu$-continuous. Hence, applying Theorem 1, (I) and (II) follow.

Corollary 3. Under the assumptions of Corollary 2, the mapping $f$ admits one and only one fixed point.

Proof. Suppose that $u, v \in \operatorname{Fix}(f)$. Since by Corollary 2 we know that $\operatorname{Fix}(f)$ is $g$ admissible, then $g(u)=g(v)=0$. Taking $(y, z)=(u, v)$ in (21), it holds that

$$
\begin{aligned}
& \delta(u, v)+g(u)+g(v) \\
& \leq F(\delta(u, u)+g(u)+g(u), \delta(u, v)+g(u)+g(v))(\delta(v, v)+g(v)+g(v)) \\
& +\sigma(\delta(u, v)+g(u)+g(v)),
\end{aligned}
$$

that is,

$$
\delta(u, v) \leq \sigma \delta(u, v)
$$

Since $\sigma<1$, we deduce that $u=v$. 
Corollary 4. Let $(M, \delta)$ be a complete metric space, $f: M \rightarrow M$, and $g: M \rightarrow[0,+\infty)$ be a lowe semi-continuous function. Suppose that

$$
\begin{aligned}
& \delta(f(y), f(z))+g(f(y))+g(f(z)) \\
& \leq \lambda\left[\frac{\tau+\delta(y, f(y))+g(y)+g(f(y))}{\tau+\delta(y, z)+g(y)+g(z)}\right](\delta(z, f(z))+g(z)+g(f(z))) \\
& +\sigma(\delta(y, z)+g(y)+g(z))
\end{aligned}
$$

for all $y, z \in M$, where $\lambda, \sigma, \tau>0$ and $\lambda+\sigma<1$. Then

(I) $\operatorname{Fix}(f)$ is $g$-admissible,

(II) For all $z_{0} \in M$, the Picard sequence $\left\{f^{n}\left(z_{0}\right)\right\}$ converges to a fixed point of $f$.

(III) $\operatorname{card}(\operatorname{Fix}(f))=1$.

Proof. We have just to observe that (22) is a special case of (21) with

$$
F(t, s)=\frac{\tau+t}{\tau+s}, \quad t, s \geq 0
$$

Next, the results follow from Corollaries 2 and 3.

Remark 2. The class of mappings $f: M \rightarrow M$ satisfying (22) with $g \equiv 0$ and $\tau=1$ has been introduced in [26] under the name of rational-type contractions. Namely, it was shown that, if $f$ belongs to this class, then $\operatorname{card}(\operatorname{Fix}(f))=1$ and for all $z_{0} \in M$, the Picard sequence $\left\{f^{n}\left(z_{0}\right)\right\}$ converges to the fixed point of $f$. Clearly, taking $g \equiv 0$ and $\tau=1$ in Corollary 4 , we refind these results. For additional references related to rational-type contractions, see, e.g., [27-30] and the references therein.

An example supporting Corollary 4 is given below.

Example 5. Let $M=[0,1]$ and $\delta$ be the metric on $M$ given by (18). Let us consider the mappings $f: M \rightarrow M$ and $g: M \rightarrow[0,+\infty)$, where $f$ is given by (19) and

$$
g(y)=\frac{5}{6} y\left|y-\frac{1}{4}\right|, \quad y \in M
$$

Obviously, $\operatorname{Fix}(f)=\left\{\frac{1}{4}\right\}$ and $g^{-1}(\{0\})=\left\{0, \frac{1}{4}\right\}$. We claim that $f$ and $g$ satisfy (22) with $\sigma=\frac{1}{2}, 0<\lambda<\frac{1}{2}$ and $\tau>0$.

- Case 1: $0 \leq y, z<1$. In this case,

$$
\delta(f(y), f(z))+g(f(y))+g(f(z))=2 g\left(\frac{1}{4}\right)=0
$$


Then (22) holds.

- Case 2: $0 \leq y<1$ and $z=1$. In this case,

$$
\begin{aligned}
& \delta(f(y), f(z))+g(f(y))+g(f(z)) \\
& =\frac{1}{4}+g\left(\frac{1}{4}\right)+g(0) \\
& =\frac{1}{4} \\
& \leq \frac{1}{2} \frac{5}{8} \\
& =\sigma g(z) \\
& \leq \sigma(\delta(y, z)+g(y)+g(z)) \\
& +\lambda\left[\frac{\tau+\delta(y, f(y))+g(y)+g(f(y))}{\tau+\delta(y, z)+g(y)+g(z)}\right](\delta(z, f(z))+g(z)+g(f(z))) .
\end{aligned}
$$

- Case 3: $0 \leq z<1$ and $y=1$. In this case

$$
\begin{aligned}
& \delta(f(y), f(z))+g(f(y))+g(f(z)) \\
& =\frac{1}{4}+g(0)+g\left(\frac{1}{4}\right) \\
& =\frac{1}{4} \\
& \leq \sigma g(y) \\
& \leq \sigma(\delta(y, z)+g(y)+g(z)) \\
& +\lambda\left[\frac{\tau+\delta(y, f(y))+g(y)+g(f(y))}{\tau+\delta(y, z)+g(y)+g(z)}\right](\delta(z, f(z))+g(z)+g(f(z))) .
\end{aligned}
$$

- Case 4: $y=z=1$. In this case

$$
\delta(f(y), f(z))+g(f(y))+g(f(z))=2 g(0)=0 .
$$

Then (22) holds. Hence, (22) is satisfied in all cases. Then, by Corollary 4, $\operatorname{Fix}(f)$ is gadmissible, and for all $z_{0} \in M$, the Picard sequence $\left\{f^{n}\left(z_{0}\right)\right\}$ converges to the fixed point of $f$. Notice that

$$
\operatorname{Fix}(f)=\left\{\frac{1}{4}\right\} \subset\left\{0, \frac{1}{4}\right\}=g^{-1}(\{0\}),
$$

which confirms the results given by Corollary 4.

\section{Conclusions}

We provided sufficient conditions under which the set of fixed points of a mapping $f: M \rightarrow M$ is included in the zero set of a mapping $g: M \rightarrow[0,+\infty)$ (see Theorems 1 and 2 ) Next, we discussed some special cases of our obtained results and provided some examples (see Section 4).

Funding: The author is supported by Researchers Supporting Project number (RSP-2021/4), King Saud University, Riyadh, Saudi Arabia.

Institutional Review Board Statement: Not applicable.

Informed Consent Statement: Not applicable.

Data Availability Statement: Not applicable.

Conflicts of Interest: The author declares no conflict of interest. 


\section{References}

1. Bukatin, M.; Kopperman, R.; Matthews, S.; Pajoohesh, H. Partial metric spaces. Am. Math. Mon. 2009, 116, 708-718. [CrossRef]

2. Matthews, S. An extensional treatment of lazy data flow deadlock. Theoret. Comput. Sci. 1995, 151, 195-205. [CrossRef]

3. Rus, I.A. Fixed point theory in partial metric spaces. Anal. Univ. Vest Timis. Ser. Mat. Inform. 2008, 46, 141-160.

4. Oltra, S.; Valero, O. Banach's fixed point theorem for partial metric spaces. Rend. Istit. Mat. Univ. Trieste. 2004, 36, 17-26.

5. Samet, B. Existence and uniqueness of solutions to a system of functional equations and applications to partial metric spaces. Fixed Point Theory. 2013, 14, 473-481.

6. Fan, Y.F.; Zhu, C.X.; Wu, Z.Q. Some $\varphi$-coupled fixed point results via modified F-control function's concept in metric spaces and its applications. J. Comput. Appl. Math. 2019, 349, 70-81. [CrossRef]

7. Imdad, M.; Khan, A.R.; Saleh, H.N.; Alfaqih, W.M. Some $\varphi$-fixed point results for $(F, \varphi, \alpha-\psi)$-contractive type mappings with applications. Mathematics 2019, 7, 122. [CrossRef]

8. Karapinar, E.; O'Regan, D.; Samet, B. On the existence of fixed points that belong to the zero set of a certain function. Fixed Point Theory Appl. 2015, 2015, 152. [CrossRef]

9. Kumrod, P.; Sintunavarat, W. A new contractive condition approach to $\varphi$-fixed point results in metric spaces and its applications. J. Comput. Appl. Math. 2017, 311, 194-204. [CrossRef]

10. Roldán-López-de-Hierro, A.F.; Samet, B. $\varphi$-admissibility results via extended simulation functions. J. Fixed Point Theory Appl. 2017, 19, 1997-2015.

11. Samet, B.; Vetro, C.; Vetro, F. From metric spaces to partial metric spaces. Fixed Point Theory Appl. 2013, 2013, 5. [CrossRef]

12. Vetro, F. Fixed point belonging to the zero-set of a given function. J. Nonlinear Sci. Appl. 2018, 11, 417-424. [CrossRef]

13. Gu, F.; Shatanawi, W. Some new results on common coupled fixed points of two hybrid pairs of mappings in partial metric spaces. J. Nonlinear Funct. Anal. 2019, 2019, 13.

14. Gunasekar, T.; Karpagam, S.; Zlatanov, B. On p-Cyclic Orbital M-K Contractions in a Partial Metric Space. Mathematics 2018, 6, 116. [CrossRef]

15. Hristov, M.; Ilchev, A.; Zlatanov, B. On the Best Proximity Points for p-Cyclic Summing Contractions. Mathematics 2020, 8, 1060. [CrossRef]

16. Petrusel, A. Local fixed point results for graphic contractions. J. Nonlinear Var. Anal. 2019, 3, 141-148.

17. Samet, B. On the approximation of fixed points for a new class of generalized Berinde mappings. Carpathian J. Math. 2016, 32, 363-374. [CrossRef]

18. Samet, B.; Vetro, C.; Vetro, P. Fixed point theorems for $(\alpha, \psi)$-contractive type mappings. Nonlinear Anal. 2012, 75, $2154-2165$. [CrossRef]

19. Berinde, V. Iterative Approximation of Fixed Points; Lecture Notes in Mathematics; Springer: Berlin/Heidelberg, Germany, 2007.

20. Berinde, V. Approximating fixed points of weak contractions using the Picard iteration. Nonlinear Anal. Forum. 2004, 9, 43-53.

21. Al-Mezel, S.A.; Ahmad, J. Generalized fixed point results for almost $\left(\alpha, F_{\sigma}\right)$-contractions with applications to Fredholm integral inclusions. Symmetry 2019, 11, 1068. [CrossRef]

22. Berinde, V. Approximating fixed points of almost convex contractions in metric spaces. Ann. Acad. Rom. Sci. Ser. Math. Appl. 2020, 12, 11-23.

23. Hammad, H.A.; De la Sen, M. Fixed-point results for a generalized almost $(s, q)$-Jaggi F-contraction-type on b-metric-like spaces. Mathematics 2020, 8, 63. [CrossRef]

24. Iqbal, I.; Batool, A.; Ege, O.; Sen, M. Fixed point of almost contraction in b-metric spaces. J. Math. 2020, 2020, 6. [CrossRef]

25. Shatanawi, W.; Postolache, M. Coincidence and fixed point results for generalized weak contractions in the sense of Berinde on partial metric spaces. Fixed Point Theory Appl. 2013, 2013, 54. [CrossRef]

26. Dass, B.K.; Gupta, S. An extension of Banach contraction principle through rational expressions. Indian J. Pure Appl. Math. 1975, 6, 1455-1458.

27. Choudhury, B.S.; Metiya, N.; Konar, P. Fixed point results for rational type contraction in partially ordered complex-valued metric spaces. Bull. Int. Math. Virtual. Inst. 2015, 5, 73-80.

28. Fulga, A. On $(\psi, \phi)$-rational contractions. Symmetry 2020, 12, 723. [CrossRef]

29. Huang, H.; Singh, Y. M.; Khan, M.S.; Radenović, S. Rational type contractions in extended b-metric spaces. Symmetry 2021, 13, 614. [CrossRef]

30. Jaggi, D.S. Some unique fixed point theorems. Indian J. Pure Appl. Math. 1977, 8, 223-230. 